PROCEEDINGS OF THE

AMERICAN MATHEMATICAL SOCIETY

Volume 126, Number 7, July 1998, Pages 2141-2149

S 0002-9939(98)04356-1

\title{
BORDISM OF TWO COMMUTING INVOLUTIONS
}

\author{
PEDRO L. Q. PERGHER
}

(Communicated by Thomas Goodwillie)

\begin{abstract}
In this paper we obtain conditions for a Whitney sum of three vector bundles over a closed manifold, $\varepsilon_{1} \oplus \varepsilon_{2} \oplus \varepsilon_{3} \rightarrow F$, to be the fixed data of a $\left(Z_{2}\right)^{2}$-action; these conditions yield the fact that if $\left(\varepsilon_{1} \oplus R\right) \oplus \varepsilon_{2} \oplus$ $\varepsilon_{3} \rightarrow F$ is the fixed data of a $\left(Z_{2}\right)^{2}$-action, where $R \rightarrow F$ is the trivial one dimensional bundle, then the same is true for $\varepsilon_{1} \oplus \varepsilon_{2} \oplus \varepsilon_{3} \rightarrow F$. The results obtained, together with techniques previously developed, are used to obtain, up to bordism, all possible $\left(Z_{2}\right)^{2}$-actions fixing the disjoint union of an even projective space and a point.
\end{abstract}

\section{INTRODUCTION}

From $[4,28.1]$ one has an exact sequence

$$
0 \rightarrow \mathcal{N}_{n}^{Z_{2}} \stackrel{F}{\rightarrow} \bigoplus_{j=0}^{n} \mathcal{N}_{n-j}(B O(j)) \stackrel{\partial}{\rightarrow} \mathcal{N}_{n-1}(B O(1)) \rightarrow 0
$$

which shows that the equivariant bordism class of an involution $(M, T)$ is determined by the bordism class of its fixed point data $\eta \rightarrow F_{T}$; additionally, it yields the fact that a real vector bundle $\eta \rightarrow F$ is fixed data of an involution if and only if $\lambda \rightarrow R P(\eta)$ bounds in $\mathcal{N}_{n-1}(B O(1))$, where $\lambda$ is the usual line bundle over the projective space bundle $R P(\eta)$. The first task of this paper is to obtain the analogue of this fact for $\left(Z_{2}\right)^{2}$-actions; specifically, given a Whitney sum of vector bundles over a closed manifold, $\varepsilon_{1} \oplus \varepsilon_{2} \oplus \varepsilon_{3} \rightarrow F$, we want to determine conditions in terms of Whitney numbers for this sum to be the fixed data of a $\left(Z_{2}\right)^{2}$-action (Theorem $1)$. The extension of the above sequence for $\left(Z_{2}\right)^{k}$-actions is the "exact sequence of bordism of $\left(\left(Z_{2}\right)^{k}, q\right)$-manifolds-bundles", introduced by Stong in [10] and which shows that the stationary point structure of a $\left(Z_{2}\right)^{k}$-action determines the bordism class. Looking at the case $k=1$, it is then natural to appeal to this sequence to handle the problem in question. For $k=2$ and $q=0$ it is

$$
0 \rightarrow \mathcal{N}_{n}\left(\left(Z_{2}\right)^{2}, 0\right) \stackrel{F}{\longrightarrow} \bigoplus \mathcal{N}_{r, s}\left(Z_{2}, 1\right) \stackrel{S}{\longrightarrow} \widehat{\mathcal{N}}_{n-1, s}\left(\left(Z_{2}\right)^{2}, 0\right) \rightarrow 0
$$

By iteration this sequence yields

$$
0 \rightarrow \mathcal{N}_{n}\left(\left(Z_{2}\right)^{2}, 0\right) \stackrel{F F}{\longrightarrow} \bigoplus \mathcal{N}_{r^{\prime}, r^{\prime \prime}, s^{\prime}, s^{\prime \prime}}\left(\left(Z_{2}\right)^{0}, 3\right) \stackrel{S}{\longrightarrow} \widehat{\mathcal{N}}_{r-1, s}\left(\left(Z_{2}\right)^{2}, 0\right) \rightarrow 0 .
$$

Received by the editors November 7, 1996 and, in revised form, December 12, 1996.

1991 Mathematics Subject Classification. Primary 57R85; Secondary 57R75.

Key words and phrases. $\left(Z_{2}\right)^{2}$-action, fixed data, bordism class, projective space bundle, Whitney number, Smith homomorphism.

The present work was partially supported by CNPq. 
Here, for a given $\left(Z_{2}\right)^{2}$-action $(M, \Phi)$, one has $F F[M, \Phi]=\left[\varepsilon_{1} \oplus \varepsilon_{2} \oplus \varepsilon_{3} \rightarrow F\right]$. Unfortunately, since iteration destroys exactness, the condition $S\left[\varepsilon_{1} \oplus \varepsilon_{2} \oplus \varepsilon_{3}\right]=0$ is a necessary but not sufficient condition for this sum be the fixed data of a $\left(Z_{2}\right)^{2}$ action. This will be bypassed simply by putting together that sequence with some additional facts extracted from [10] and [9]. In a certain sense, the mentioned conditions serve as a complement of [6] and [5] in the case $k=2$; in these works it was shown how to construct the equivariant bordism class of a manifold with $\left(Z_{2}\right)^{k}$-action from its fixed data.

Next we recall from [4] that for a given vector bundle $\eta \rightarrow F$ we have

$$
\Delta[\lambda \rightarrow R P(\eta \oplus R)]=[\lambda \rightarrow R P(\eta)],
$$

where $\Delta: \mathcal{N}_{n}(B O(1)) \rightarrow \mathcal{N}_{n-1}(B O(1))$ is the Smith homomorphism and $R \rightarrow F$ denotes the trivial 1-dimensional bundle over $F$. This yields particularly the fact that if $\eta \oplus R \rightarrow F$ is fixed data of an involution, then the same is true for $\eta \rightarrow F$. The second step of this paper will consist of using the above conditions to obtain the analogue of this fact for $\left(Z_{2}\right)^{2}$-actions; specifically, we will show that if $\varepsilon_{1}, \varepsilon_{2}$ and $\varepsilon_{3}$ are vector bundles over $F$ such that $\left(\varepsilon_{1} \oplus R\right) \oplus \varepsilon_{2} \oplus \varepsilon_{3} \rightarrow F$ is fixed data of some $\left(Z_{2}\right)^{2}$-action, then the same is valid for $\varepsilon_{1} \oplus \varepsilon_{2} \oplus \varepsilon_{3} \rightarrow F$ (Theorem 2).

Finally, together with facts of [7], the above result will be used to obtain, up to bordism, all possible $\left(Z_{2}\right)^{2}$-actions fixing $R P(2 n) \cup\{p\}, p=$ point (Theorem 3 ). Referring to this we observe that in [7] we have developed a method to analyse the bordism classes of $\left(Z_{2}\right)^{k}$-actions fixing $V^{n} \cup\{p\}$, where $V^{n}$ is a connected closed $n$-dimensional manifold, and where the starting point is the knowledge of the set $\mathcal{A}$ of all equivariant bordism classes of involutions containing a representative $(M, T)$ with $M$ connected and with the above fixed set. This method has shown itself particularly effective when $\mathcal{A}$ has a single element; in this case, if $(M, \Phi)$ is a $\left(Z_{2}\right)^{k}$ action fixing $V^{n} \cup\{p\}$, we have seen that the fixed data of $(M, \Phi)$ bears, in terms of bordism, a strong resemblance to the fixed data of $\sigma \Gamma_{t}^{k}(W, \tau)$, where $[(W, \tau)]$ is the only element of $\mathcal{A}$ and $\sigma \Gamma_{t}^{k}$ denotes certain operations which produce special $\left(Z_{2}\right)^{k}$ actions from a given involution. In particular, we have proved further that $(M, \Phi)$ is bordant to some action of type $\sigma \Gamma_{t}^{k}(W, \tau)$ when $V^{n}=S^{p} \times S^{q}, S^{n}$ or $R P(2 p+1)$ (see [7] and [8]). However, if $\mathcal{A}$ has more than one element, the classification seems more difficult because of the fact that the results of [7] indicate the possibility of occurrence of other classes added to those produced by the operations $\sigma \Gamma_{t}^{k}$. In fact, as it will be seen, this is what surprisingly happens when $V^{n}=R P(2 r)$ (one knows from [3] that in this case the set $\mathcal{A}$ has more than one element). So in this case the results of [7] are not definitively enough to determine the classification. In this way the above results constitute, at least in the case $k=2$, an additional tool to handle this question.

\section{Conditions For a Whitney sum of VeCtor Bundles TO BE THE FIXED DATA OF A $\left(Z_{2}\right)^{2}$-ACTION}

As usual, $\left(Z_{2}\right)^{2}$ is considered as the group generated by two commuting involutions $T_{1}, T_{2}$. Given a $\left(Z_{2}\right)^{2}$-action $(M, \Phi), \Phi=\left(T_{1}, T_{2}\right)$, one may consider the fixed data of $\Phi$ as being $\varepsilon_{1} \oplus \varepsilon_{2} \oplus \varepsilon_{3} \rightarrow F_{\Phi}$, where $F_{\Phi}$ denotes the set of stationary points of $\Phi$ and $\varepsilon_{1}, \varepsilon_{2}$ and $\varepsilon_{3}$ are the normal bundles of $F_{\Phi}$ in $F_{T_{2}}, F_{T_{1} T_{2}}$ and $F_{T_{1}}$, respectively (this establishes a standard order for the fixed data which can be naturally extended for $\left(Z_{2}\right)^{k}$-actions). We will be using $\lambda_{i}, i=1,2,3$, to denote the line 
bundle over $R P\left(\varepsilon_{i}\right)$ (or occasionally over $R P\left(\varepsilon_{i} \oplus R\right)$ ) and will also be suppressing all bundle maps.

One starts then with a Whitney sum of real vector bundles over a closed manifold, $\varepsilon_{1} \oplus \varepsilon_{2} \oplus \varepsilon_{3} \rightarrow F$, and wishes that this sum be the fixed data of a $\left(Z_{2}\right)^{2}$-action $\left(M^{n}, \Phi\right)$. Here $F$ is not assumed to be connected and for each component $F_{i}$ one must have

$$
\operatorname{dim}\left(\varepsilon_{1 \mid F_{i}}\right)+\operatorname{dim}\left(\varepsilon_{2 \mid F_{i}}\right)+\operatorname{dim}\left(\varepsilon_{3 \mid F_{i}}\right)+\operatorname{dim}\left(F_{i}\right)=n
$$

For each $0 \leq l \leq n$ one has the Stong's sequence of $\left(Z_{2}, 1\right)$-manifolds-bundles before mentioned:

$$
0 \rightarrow \mathcal{N}_{l, n-l}\left(Z_{2}, 1\right) \stackrel{F_{l}}{\longrightarrow} \bigoplus_{l^{\prime}, l^{\prime \prime}, g^{\prime}, g^{\prime \prime}} \mathcal{N}_{l^{\prime}, l^{\prime \prime}, g^{\prime}, g^{\prime \prime}}\left(Z_{2}^{0}, 3\right) \stackrel{S_{l}}{\longrightarrow} \widehat{\mathcal{N}}_{l-1, n-l}\left(Z_{2}, 1\right) \rightarrow 0
$$

where the sum is over all sequences with $l^{\prime}+l^{\prime \prime}=l, g^{\prime}+g^{\prime \prime}=n-l$. Taking the sum over $0 \leq l \leq n$ one obtains the exact sequence

$$
\begin{aligned}
0 \rightarrow \bigoplus_{l} \mathcal{N}_{l, n-l}\left(Z_{2}, 1\right) \stackrel{\oplus F_{l}}{\longrightarrow} \bigoplus_{l} \bigoplus_{l^{\prime}, l^{\prime \prime}, g^{\prime}, g^{\prime \prime}} \mathcal{N}_{l^{\prime}, l^{\prime \prime}, g^{\prime}, g^{\prime \prime}}\left(Z_{2}^{0}, 3\right) \\
\stackrel{\oplus S_{l}}{\longrightarrow} \bigoplus_{l} \widehat{\mathcal{N}}_{l-1, n-l}\left(Z_{2}, 1\right) \rightarrow 0
\end{aligned}
$$

and $\varepsilon_{1} \oplus \varepsilon_{2} \oplus \varepsilon_{3} \rightarrow F$ represents a bordism class $\alpha$ of the middle term. Suppose $\oplus S_{l}(\alpha)=0$; as it can be seen by using the definition of $S_{l}$ and $[9,8.7]$, this means that $\lambda_{1} \oplus\left(\varepsilon_{2} \oplus\left(\varepsilon_{3} \otimes \lambda_{1}\right)\right) \rightarrow R P\left(\varepsilon_{1}\right)$ bounds as an element of

$$
\bigoplus_{l} \mathcal{N}_{l-1}(B O(1) \times B O(n-l)) .
$$

Then there is $\beta \in \bigoplus_{l} \mathcal{N}_{l, n-l}\left(Z_{2}, 1\right)$ such that $\oplus F_{l}(\beta)=\alpha ; \beta$ is the bordism class of a 4-tuple $(V, T ; \eta, \bar{T})$, where $T$ is an involution on the closed manifold $V, \eta$ is a vector bundle over $V$ and $\bar{T}$ is an involution of $\eta$ by a bundle map covering $T$ (we will be omitting the indication about the variation of dimension over the several components, but this should always be assumed). According to a remark after Proposition 8 of [9, page 67] one can exhibit an explicit representative for $\beta$ : denoting a typical element of the total space of the bundle $\varepsilon_{2} \oplus\left(\varepsilon_{3} \otimes \lambda_{1}\right) \rightarrow$ $R P\left(\varepsilon_{1} \oplus R\right)$ by $\left((u, w \otimes r),[v, s]_{p}\right)$, where $(u, w \otimes r)$ belongs to the fiber of $\varepsilon_{2} \oplus\left(\varepsilon_{3} \otimes \lambda_{1}\right)$ over $[v, s]_{p}$, which in turn denotes an element of the total space of $R P\left(\varepsilon_{1} \oplus R\right)$ belonging to the fiber over the point $p \in F$, the correspondence

$$
\left((u, w \otimes r),[v, s]_{p}\right) \rightarrow\left((u,-w \otimes r),[-v, s]_{p}\right)
$$

defines an involution $T$ on the total space of $\varepsilon_{2} \oplus\left(\varepsilon_{3} \otimes \lambda_{1}\right)$ recovering the involution $t$ on $R P\left(\varepsilon_{1} \oplus R\right)$ given by $t[v, s]_{p}=[-v, s]_{p}$. The mentioned representative is then

$$
\left(R P\left(\varepsilon_{1} \oplus R\right), t ; \varepsilon_{2} \oplus\left(\varepsilon_{3} \otimes \lambda_{1}\right), T\right) .
$$

Considering the Stong's sequence relative to $k=2, q=0$,

$$
0 \rightarrow \mathcal{N}_{n}\left(\left(Z_{2}\right)^{2}, 0\right) \stackrel{F}{\longrightarrow} \bigoplus_{l} \mathcal{N}_{l, n-l}\left(Z_{2}, 1\right) \stackrel{S}{\longrightarrow} \widehat{\mathcal{N}}_{n-1}\left(\left(Z_{2}\right)^{2}, 0\right) \rightarrow 0
$$

all that remains is to analyse more closely the condition $S(\beta)=0$; in fact, this condition will guarantee the existence of a $\left(Z_{2}\right)^{2}$-action $\left(M^{n}, \Phi\right)$ with $\bigoplus_{l} F_{l}\left(F\left[M^{n}, \Phi\right]\right)=$ 
$\alpha$, that is, with fixed data bordant to $\varepsilon_{1} \oplus \varepsilon_{2} \oplus \varepsilon_{3} \rightarrow F$. But a standard equivariant construction yields in this case a $\left(Z_{2}\right)^{2}$-action having precisely this fixed data.

We remark that $\widehat{\mathcal{N}}_{n-1}\left(\left(Z_{2}\right)^{2}, 0\right)$ is the bordism group of $\left(Z_{2}\right)^{2}$-actions for which the first involution acts freely. Using the definition of $S$ we see that a representative of $S(\beta)$ is $\left(S\left(\varepsilon_{2} \oplus\left(\varepsilon_{3} \otimes \lambda_{1}\right)\right), A, T\right)$, where $S()$ means sphere bundle construction, $A$ is the antipodal involution and $T$ is restriction of the previous $T$. Now one knows from [10, Prop.3] that $S$ maps the summand $\mathcal{N}_{n-1,1}\left(Z_{2}, 1\right)$ isomorphically onto $\widehat{\mathcal{N}}_{n-1}\left(\left(Z_{2}\right)^{2}, 0\right)$ and that the inverse for $S$ on this summand is given by

$$
\left[\left(W ; T_{1}, T_{2}\right)\right] \rightarrow\left[\left(W / T_{1}, T_{2}^{*} ; \lambda, \bar{T}_{2}^{*}\right)\right]
$$

where $\lambda$ is the line bundle of the double cover of $W / T_{1}$ by $W$ and $T_{2}^{*}, \bar{T}_{2}^{*}$ are induced by $T_{2}$. Hence we must analyse the class of

$$
\left(R P\left(\varepsilon_{2} \oplus\left(\varepsilon_{3} \otimes \lambda_{1}\right)\right), T^{*} ; \lambda^{\prime}, \bar{T}^{*}\right)
$$

in $\mathcal{N}_{n-1,1}\left(Z_{2}, 1\right)$, where $\lambda^{\prime}$ is the line bundle over $R P\left(\varepsilon_{2} \oplus\left(\varepsilon_{3} \otimes \lambda_{1}\right)\right.$ ) (which fibers over $\left.R P\left(\varepsilon_{1} \oplus R\right)\right)$ and $T^{*}, \bar{T}^{*}$ are, as above described, induced by the previous $T$. To make this analysis one simply looks at the fixed point information; according to [10, Prop. 3], for a given element $[(W, T ; \eta, \bar{T})] \in \mathcal{N}_{l, p}\left(Z_{2}, 1\right)$, this information is given by the class of $\mu \oplus \eta^{+} \oplus \eta^{-} \rightarrow F_{T}$ in

$$
\bigoplus_{i=0}^{l} \bigoplus_{p^{\prime}+p^{\prime \prime}=p} \mathcal{N}_{i}\left(B O(l-i) \times B O\left(p^{\prime}\right) \times B O\left(p^{\prime \prime}\right)\right)
$$

where $\mu \rightarrow F_{T}$ is the normal bundle of $F_{T}$ in $W$ and $\eta_{\mid F_{T}}=\eta^{+} \oplus \eta^{-}, \eta^{+}$and $\eta^{-}$ denoting the subbundles of $\eta_{\mid F_{T}}$ where $\bar{T}$ acts as +1 and -1 , respectively. by

Adopting the type of notation previously established one sees that $\bar{T}^{*}$ is given

$$
\left(\left[(u, w \otimes r),[v, s]_{p}\right], x\right) \rightarrow\left(\left[(u,-w \otimes r),[-v, s]_{p}\right], x\right) .
$$

The fixed set of $T^{*}$ must be taken over the fixed set of $t$, which is the disjoint union of $R P\left(\varepsilon_{1}\right)$ and $R P(R)=F$.

1)Over $R P\left(\varepsilon_{1}\right)$ one has

$$
\begin{array}{r}
T^{*}\left[(u, w \otimes r),[v, 0]_{p}\right]=\left[(u,-w \otimes r),[-v, 0]_{p}\right]=\left[(u,-w \otimes-r),[v, 0]_{p}\right] \\
=\left[(u, w \otimes r),[v, 0]_{p}\right],
\end{array}
$$

that is, $T^{*}$ acts trivially. Hence $R P\left(\varepsilon_{2} \oplus\left(\varepsilon_{3} \otimes \lambda_{1}\right)\right)$ fibering over $R P\left(\varepsilon_{1}\right)$ is a component of the fixed set of $T^{*}$, and the normal bundle is

$$
\lambda_{1} \rightarrow R P\left(\varepsilon_{2} \oplus\left(\varepsilon_{3} \otimes \lambda_{1}\right)\right) .
$$

Over this component $\bar{T}^{*}$ acts trivially, so $\eta^{+} \oplus \eta^{-}$is $\lambda^{\prime} \oplus 0$ restricted to this component.

2)Over $R P(R)=F$ one has $\lambda_{1}=R$, hence $R P\left(\varepsilon_{2} \oplus\left(\varepsilon_{3} \otimes \lambda_{1}\right)\right)=R P\left(\varepsilon_{2} \oplus \varepsilon_{3}\right)$; further, $T^{*}$ acts in this case as

$$
\left[(u, w \otimes r),[0, s]_{p}\right] \rightarrow\left[(u,-(w \otimes r)),[0, s]_{p}\right] .
$$

Therefore one has two components of fixed points:

a) $R P\left(\varepsilon_{2}\right)$ fibering over $F$ with normal bundle $\left(\varepsilon_{3} \otimes \lambda_{2}\right) \oplus \varepsilon_{1}$;

b) $R P\left(\varepsilon_{3}\right)$ fibering over $F$ with normal bundle $\left(\varepsilon_{2} \otimes \lambda_{3}\right) \oplus \varepsilon_{1}$. 
Over the component of a) $\bar{T}^{*}$ acts trivially and $\lambda^{\prime}=\lambda_{2}$, hence $\eta^{+} \oplus \eta^{-}=\lambda_{2} \oplus 0$. Over the component of $\mathrm{b}$ ) one has

$$
\bar{T}^{*}\left(\left[(0, w \otimes r),[0, s]_{p}\right], x\right)=\left(\left[(0,-w \otimes r),[0, s]_{p}\right], x\right)=\left(\left[(0, w \otimes r),[0, s]_{p}\right],-x\right)
$$

and $\lambda^{\prime}=\lambda_{3}$, hence $\eta^{+} \oplus \eta^{-}=0 \oplus \lambda_{3}$.

One concludes that $S(\beta)=0$ if and only if

$$
\begin{aligned}
\left(\lambda_{1} \oplus \lambda^{\prime} \oplus 0 \rightarrow R P\left(\varepsilon_{2} \oplus\left(\varepsilon_{3} \otimes \lambda_{1}\right)\right)\right) & \cup\left(\left(\left(\varepsilon_{3} \otimes \lambda_{2}\right) \oplus \varepsilon_{1}\right) \oplus \lambda_{2} \oplus 0 \rightarrow R P\left(\varepsilon_{2}\right)\right) \\
& \cup\left(\left(\left(\varepsilon_{2} \otimes \lambda_{3}\right) \oplus \varepsilon_{1}\right) \oplus 0 \oplus \lambda_{3} \rightarrow R P\left(\varepsilon_{3}\right)\right)
\end{aligned}
$$

bounds in

$$
\begin{aligned}
& \left(\bigoplus_{l=0}^{n-1} \mathcal{N}_{l}(B O(n-1-l) \times B O(1) \times B O(0))\right) \\
& \quad \oplus\left(\bigoplus_{l=0}^{n-1} \mathcal{N}_{l}(B O(n-1-l) \times B O(0) \times B O(1))\right),
\end{aligned}
$$

where the first base space fibers over $R P\left(\varepsilon_{1}\right)$ and the remaining fiber over $F$ (note that no contribution is made by the components $F_{i}$ with dimension=n, which can be ignored with no loss). Putting together with $\oplus S_{l}(\alpha)=0$ and taking into account the dimension of the bordism groups involved, one obtains the following

Theorem 1. A Whitney sum $\varepsilon_{1} \oplus \varepsilon_{2} \oplus \varepsilon_{3} \rightarrow F$ is fixed data of a $\left(Z_{2}\right)^{2}$-action if and only if the following conditions hold:

a) $\lambda_{1} \oplus\left(\varepsilon_{2} \oplus\left(\varepsilon_{3} \otimes \lambda_{1}\right)\right) \rightarrow R P\left(\varepsilon_{1}\right)$ bounds in $\bigoplus_{l=0}^{n-1} \mathcal{N}_{l}(B O(1) \times B O(n-1-l))$, where $\operatorname{RP}\left(\varepsilon_{1}\right)$ fibers over $F$;

b) $\left(\lambda_{1} \oplus \lambda^{\prime} \oplus 0 \rightarrow R P\left(\varepsilon_{2} \oplus\left(\varepsilon_{3} \otimes \lambda_{1}\right)\right)\right) \cup\left(\left(\left(\varepsilon_{3} \otimes \lambda_{2}\right) \oplus \varepsilon_{1}\right) \oplus \lambda_{2} \oplus 0 \rightarrow R P\left(\varepsilon_{2}\right)\right)$ bounds in $\bigoplus_{l=0}^{n-1} \mathcal{N}_{l}(B O(n-1-l) \times B O(1) \times B O(0))$, where the base spaces fiber over $R P\left(\varepsilon_{1}\right)$ and $F$, respectively;

c) $\left(\left(\varepsilon_{2} \otimes \lambda_{3}\right) \oplus \varepsilon_{1}\right) \oplus 0 \oplus \lambda_{3} \rightarrow R P\left(\varepsilon_{3}\right)$ bounds in

$$
\bigoplus_{l=0}^{n-1} \mathcal{N}_{l}(B O(n-1-l) \times B O(0) \times B O(1))
$$

with $R P\left(\varepsilon_{3}\right)$ fibering over $F$.

Since the conditions obtained are detectable through Whitney numbers calculation, our first purpose is established.

Remark. Looking closer at the dimensions of the bordism groups involved, one observes that condition (b) can alternatively be replaced by the two following conditions:

b1)

$$
\begin{aligned}
& \left(\lambda_{1} \oplus \lambda^{\prime} \oplus 0 \rightarrow R P\left(\varepsilon_{2} \oplus\left(\varepsilon_{3} \otimes \lambda_{1}\right)\right)\right) \\
& \quad \cup\left(\bigcup_{\operatorname{dim}\left(\varepsilon_{1}\right)+\operatorname{dim}\left(\varepsilon_{3}\right)=1}\left(\left(\varepsilon_{3} \otimes \lambda_{2}\right) \oplus \varepsilon_{1}\right) \oplus \lambda_{2} \oplus 0 \rightarrow R P\left(\varepsilon_{2}\right)\right)
\end{aligned}
$$

bounds in $\mathcal{N}_{n-2}(B O(1) \times B O(1) \times B O(0))$; 
b2)

$$
\begin{gathered}
\bigcup_{\operatorname{dim}\left(\varepsilon_{1}\right)+\operatorname{dim}\left(\varepsilon_{3}\right) \neq 1}\left(\left(\varepsilon_{3} \otimes \lambda_{2}\right) \oplus \varepsilon_{1}\right) \oplus \lambda_{2} \oplus 0 \rightarrow R P\left(\varepsilon_{2}\right) \\
\text { bounds in } \bigoplus_{l=0}^{n-1} \mathcal{N}_{l}(B O(n-1-l) \times B O(1) \times B O(0)) .
\end{gathered}
$$

\section{A teChNicAl RESUlt}

The result to be proved in this section is:

Theorem 2. Suppose that $\varepsilon_{1}, \varepsilon_{2}$ and $\varepsilon_{3}$ are vector bundles over $F$ such that $\left(\varepsilon_{1} \oplus\right.$ $R) \oplus \varepsilon_{2} \oplus \varepsilon_{3} \rightarrow F$ is fixed data of a $\left(Z_{2}\right)^{2}$-action. Then $\varepsilon_{1} \oplus \varepsilon_{2} \oplus \varepsilon_{3} \rightarrow F$ is fixed data of a $\left(Z_{2}\right)^{2}$-action.

Proof. It is easy to check that if a Whitney sum of the form $R \oplus \varepsilon \oplus 0$ is fixed data of a $\left(Z_{2}\right)^{2}$-action, then $\lambda \rightarrow R P(\varepsilon)$ bounds; hence we can assume $\operatorname{dim}\left(\varepsilon_{1}\right)+$ $\operatorname{dim}\left(\varepsilon_{3}\right)>0$, that is, $\operatorname{dim}\left(\varepsilon_{1} \oplus R\right)+\operatorname{dim}\left(\varepsilon_{3}\right)>1$. But then the remark after Theorem 1 implies that

i) $\lambda_{1} \oplus\left(\varepsilon_{2} \oplus\left(\varepsilon_{3} \otimes \lambda_{1}\right)\right) \rightarrow R P\left(\varepsilon_{1} \oplus R\right)$,

ii) $\lambda_{1} \oplus \lambda^{\prime} \oplus 0 \rightarrow R P\left(\varepsilon_{2} \oplus\left(\varepsilon_{3} \otimes \lambda_{1}\right)\right)$,

iii) $\left(\left(\varepsilon_{3} \otimes \lambda_{2}\right) \oplus\left(\varepsilon_{1} \oplus R\right)\right) \oplus \lambda_{2} \oplus 0 \rightarrow R P\left(\varepsilon_{2}\right)$ and

iv) $\left(\left(\varepsilon_{2} \otimes \lambda_{3}\right) \oplus\left(\varepsilon_{1} \oplus R\right)\right) \oplus 0 \oplus \lambda_{3} \rightarrow R P\left(\varepsilon_{3}\right)$

bound in the appropriate bordism groups, with ii) fibering over $R P\left(\varepsilon_{1} \oplus R\right)$ and the remaining over $F$. From [9, Section 8 , Lemma 4$]$ one has a commutative diagram

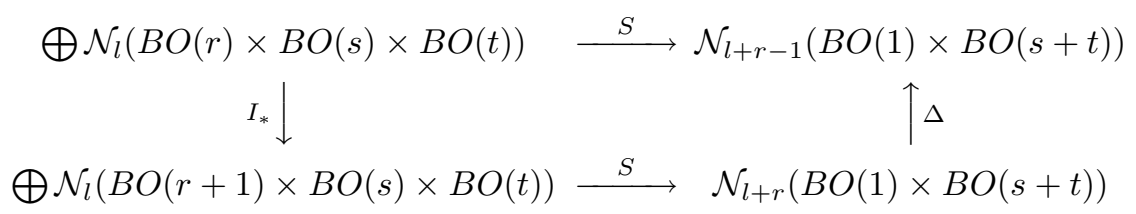

where $I_{*}$ adds a trivial line bundle to the first factor, $\Delta$ is the Smith homomorphism and the sum is over $(l, r, s, t)$ with $l+r+s+t=n$ and $0 \leq l \leq n-1$. Since $S I^{*}\left[\varepsilon_{1} \oplus \varepsilon_{2} \oplus \varepsilon_{3}\right]$ is the class of i), the class obtained from i) by replacing $\varepsilon_{1} \oplus R$ by $\varepsilon_{1}$ is zero. Now the fact that the classes obtained from iii) and iv) by replacing $\varepsilon_{1} \oplus R$ by $\varepsilon_{1}$ are also zero can be checked trivially by looking at the characteristic classes. Noting that if the classes obtained from i), ii), iii) and iv) by replacing $\varepsilon_{1} \oplus R$ by $\varepsilon_{1}$ are zero, then conditions (a), (b) and (c) of Theorem 1 will be valid for $\varepsilon_{1} \oplus \varepsilon_{2} \oplus \varepsilon_{3}$, all that remains is to prove the following

Lemma. Let $\eta \rightarrow F$ and $\mu \rightarrow R P(\eta \oplus R)$ be vector bundles such that $\xi \oplus \lambda \rightarrow R P(\mu)$ bounds in $\mathcal{N}_{*}(B O(1) \times B O(1))$, where $\xi \rightarrow R P(\mu)$ is the line bundle and $\lambda \rightarrow R P(\mu)$ is the pullback of the line bundle $\lambda \rightarrow R P(\eta \oplus R)$. Then $\xi^{\prime} \oplus \lambda^{\prime} \rightarrow R P\left(\mu^{\prime}\right)$ bounds in $\mathcal{N}_{*-1}(B O(1) \times B O(1))$; here, $\mu^{\prime}=\mu_{\mid R P(\eta)}, \xi^{\prime}=\xi_{\mid R P\left(\mu^{\prime}\right)}$ and $\lambda^{\prime}=\lambda_{\mid R P\left(\mu^{\prime}\right)}$.

Proof. Denote the total Stiefel-Whitney class of $\eta$ by $W(\eta)=1+v_{1}+v_{2}+\ldots+v_{r}$ and let $c, c^{\prime}, d$ and $d^{\prime}$ be, respectively, the characteristic classes of $\lambda, \lambda^{\prime}, \xi$ and $\xi^{\prime}$. Let $j: R P\left(\mu^{\prime}\right) \rightarrow R P(\mu)$ be the inclusion and $\theta$ be the vector bundle tangent to the fibers of $R P(\mu) \rightarrow R P(\eta \oplus R)$; set $\theta^{\prime}=\theta_{\mid R P\left(\mu^{\prime}\right)}$. Denote yet $W(R P(\mu))=1+W_{1}+$ $W_{2}+\ldots+W_{n}, W\left(R P\left(\mu^{\prime}\right)\right)=1+w_{1}+w_{2}+\ldots+w_{n-1}, W(\theta)=1+L_{1}+L_{2}+\ldots+L_{s}$, 
$W\left(\theta^{\prime}\right)=1+L_{1}^{\prime}+L_{2}^{\prime}+\ldots+L_{s}^{\prime}$. Clearly $j^{*}(c)=c^{\prime}, j^{*}(d)=d^{\prime}$ and $j^{*}\left(L_{i}\right)=L_{i}^{\prime}$. From [1] one has

$$
W\left(R P\left(\mu^{\prime}\right)\right)=W(F)\left(\sum_{i=0}^{r}\left(1+c^{\prime}\right)^{r-i} v_{i}\right)\left(1+L_{1}^{\prime}+\ldots+L_{s}^{\prime}\right)
$$

and

$$
W(R P(\mu))=(1+c) W(F)\left(\sum_{i=0}^{r}(1+c)^{r-i} v_{i}\right)\left(1+L_{1}+\ldots+L_{s}\right)
$$

where the classes $v_{i}, W(F) \in H^{*}\left(F, Z_{2}\right)$ are being considered, with the same notation, as classes in $H^{*}(R P(\mu))$ or $H^{*}\left(R P\left(\mu^{\prime}\right)\right)$ via bundle maps. Hence $j^{*}\left(W_{i}\right)=$ $w_{i}+c^{\prime} w_{i-1}$ for $1 \leq i \leq n$. We will show that all Whitney numbers

$$
c^{\prime p} d^{\prime q} w_{i_{1}} w_{i_{2}} \ldots w_{i_{t}}\left[R P\left(\mu^{\prime}\right)\right]
$$

are zero; this will be made by using induction on $l=i_{1}+i_{2}+\ldots+i_{t}$. For $l=0$ one has

$$
c^{\prime p} d^{q}\left[R P\left(\mu^{\prime}\right)\right]=j^{*}\left(c^{p} d^{q}\right)\left[R P\left(\mu^{\prime}\right)\right]=c^{p} d^{q} j_{*}\left(\left[R P\left(\mu^{\prime}\right)\right]\right)=c^{p+1} d^{q}[R P(\mu)]=0
$$

by hypothesis; here we used the fact that $R P\left(\mu^{\prime}\right)$ is the submanifold of $R P(\mu)$ dual to $c$. Suppose the fact true for all $l<i_{1}+i_{2}+\ldots+i_{t}$; then

$$
\begin{array}{r}
c^{\prime p} d^{\prime q} w_{i_{1}} \ldots w_{i_{t}}\left[R P\left(\mu^{\prime}\right)\right]=c^{\prime p} d^{\prime q}\left(j^{*}\left(W_{i_{1}}\right)+c^{\prime} w_{i_{1}-1}\right) \ldots\left(j^{*}\left(W_{i_{t}}\right)+c^{\prime} w_{i_{t}-1}\right)\left[R P\left(\mu^{\prime}\right)\right] \\
=c^{\prime p} d^{\prime q} j^{*}\left(W_{i_{1}}\right) \ldots j^{*}\left(W_{i_{t}}\right)\left[R P\left(\mu^{\prime}\right)\right]+A=c^{p+1} d^{q} W_{i_{1}} \ldots W_{i_{t}}[R P(\mu)]+A=A
\end{array}
$$

by hypothesis; here $A$ is a sum of terms of the form

$$
c^{\prime a} d^{\prime q} j^{*}\left(W_{b_{1}}\right) \ldots j^{*}\left(W_{b_{e}}\right) w_{f_{1}} \ldots w_{f_{g}}\left[R P\left(\mu^{\prime}\right)\right]
$$

with $b_{1}+\ldots+b_{e}+f_{1}+\ldots+f_{g}<i_{1}+\ldots+i_{t}$. The replacement of each $j^{*}\left(W_{b_{i}}\right)$ by $w_{b_{i}}+c^{\prime} w_{b_{i}-1}$ converts $A$ to a sum of terms of the form $c^{\prime m} d^{\prime q} w_{h_{1}} \ldots w_{h_{u}}\left[R P\left(\mu^{\prime}\right)\right]$ with $h_{1}+\ldots+h_{u}<i_{1}+\ldots+i_{t}$, and all these terms are zero by the induction hypothesis.

Remark. We observe that if $\varepsilon_{1} \oplus \varepsilon_{2} \oplus \varepsilon_{3} \rightarrow F$ is fixed data of a $\left(Z_{2}\right)^{2}$-action, then the same is true for any permutation of the $\varepsilon_{i^{\prime}}$; it is then irrelevant, in Theorem 2 , to which factor $\varepsilon_{i}$ the trivial line bundle is added.

\section{4. $\left(Z_{2}\right)^{2}$-ACTIONS FIXING $R P(2 n) \cup\{p\}$}

As announced in the introduction, we will obtain in this section, up to bordism, all possible $\left(Z_{2}\right)^{2}$-actions fixing $R P(2 n) \cup\{p\}$.

The methods of [7] yield particularly specific information about $\left(Z_{2}\right)^{2}$-actions; we need first to describe this information. For a fixed smooth closed connected $n$-dimensional manifold $V^{n}$, denote by $\mathcal{A}$ the collection of all equivariant bordism classes of involutions containing a representative $(W, T)$ with $W$ connected and $V^{n} \cup\{p\}$ as fixed point set. Setting $\mathcal{A}=\left\{\left[W_{i}^{n_{i}}, \tau_{i}\right]\right\}_{i}$, let $\eta_{i} \rightarrow V^{n}$ denote the normal bundle of $V^{n}$ in each $W_{i}^{n_{i}}$. The results of [7] yield then the following

Fact 1. Let $(M, \Phi)$ be a $\left(Z_{2}\right)^{2}$-action with fixed set $V^{n} \cup\{p\}$, and let

$$
\left(\bigoplus_{j=1}^{3} \varepsilon_{j} \rightarrow V^{n}\right) \cup\left(\bigoplus_{j=1}^{3} \mu_{j} \rightarrow p\right)
$$


denote the fixed data of $\Phi$. Then one of the following situations occurs:

i)there is exactly one bundle $\varepsilon_{j_{0}}$ bordant to some $\eta_{i}$, and the remaining $\varepsilon_{j}$ 's are the 0-dimensional bundle; in this case, $\mu_{j_{0}}$ is the trivial $n_{i}$-dimensional bundle $R^{n_{i}} \rightarrow p$, and the remaining $\mu_{j}$ 's are the 0-bundle.

ii)there are exactly two bundles $\varepsilon_{j_{1}}, \varepsilon_{j_{2}}$ bordant to $\eta_{i}$ 's, and the remaining $\varepsilon_{j_{0}}$ is bordant to the tangent bundle $T\left(V^{n}\right) \rightarrow V^{n}$; in this case, considering $\varepsilon_{j_{1}}$ and $\varepsilon_{j_{2}}$ bordant, respectively, to $\eta_{i_{1}}$ and $\eta_{i_{2}}$, one has $\mu_{j_{1}}=R^{n_{i_{1}}} \rightarrow p, \mu_{j_{2}}=R^{n_{i_{2}}} \rightarrow p$ and $\mu_{j_{0}}=0$.

Next we focus our attention on the collection $\mathcal{A}$ relative to $V=R P(2 n)$. Consider the standard endomorphism $\Gamma: \mathcal{N}_{*}^{Z_{2}} \rightarrow \mathcal{N}_{*}^{Z_{2}}$ of degree one (see, for example, [4])) and the augmentation $\varepsilon: \mathcal{N}_{*}^{Z_{2}} \rightarrow \mathcal{N}_{*}^{*}$; consider yet the involution $\left(R P(2 n+1), \tau_{0}\right)$, where $\tau_{0}\left[x_{0}, x_{1}, \ldots, x_{2 n+1}\right]=\left[-x_{0}, x_{1}, \ldots, x_{2 n+1}\right]$. The fixed data of this involution is $(\lambda \rightarrow R P(2 n)) \cup\left(R^{2 n+1} \rightarrow p\right)$, where $\lambda$ is the canonical line bundle. Since $R P(2 n) \cup\{p\}$ does not bound, it follows from the strengthened Boardman $\frac{5}{2}$-theorem of [2] that there exists $k_{n} \in Z^{+}$such that $\varepsilon \Gamma^{k_{n}}\left[R P(2 n+1), \tau_{0}\right] \neq 0$ and $\varepsilon \Gamma^{i}\left[R P(2 n+1), \tau_{0}\right]=0$ for all $0 \leq i<k_{n}$. Then for each $0 \leq i \leq k_{n}$ the fixed data of $\Gamma^{i}\left(R P(2 n+1), \tau_{0}\right)$ can be considered, with no loss, as being $\left(\lambda \oplus R^{i} \rightarrow R P(2 n)\right) \cup\left(R^{2 n+i+1} \rightarrow p\right)$. In [3] Royster proved the following

Fact 2. Let $(W, T)$ be an involution fixing $R P(2 n) \cup\{p\}$. Then

$$
[W, T]=\Gamma^{i}\left[R P(2 n+1), \tau_{0}\right]
$$

for some $0 \leq i \leq k_{n}$.

Suppose now that $(M, \Phi)$ is a $\left(Z_{2}\right)^{2}$-action fixing $R P(2 n) \cup\{p\}$, and let

$$
\left(\bigoplus_{j=1}^{3} \varepsilon_{j} \rightarrow R P(2 n)\right) \cup\left(\bigoplus_{j=1}^{3} \mu_{j} \rightarrow p\right)
$$

denote the fixed data. Combining Facts 1 and 2 one has that one of the following situations occurs:

i)there is $0 \leq i \leq k_{n}$ such that, up to permutation, $\varepsilon_{1}$ is bordant to $\lambda \oplus R^{i}$ and $\varepsilon_{2}, \varepsilon_{3}$ are the 0 -bundle;

ii)there are $0 \leq i, j \leq k_{n}, i \leq j$, such that, up to permutation, $\varepsilon_{1}, \varepsilon_{2}$ and $\varepsilon_{3}$ are bordant, respectively, to $\lambda \oplus R^{i}, \lambda \oplus R^{j}$ and $T(R P(2 n))$.

But a bundle $\eta \rightarrow R P(2 n)$ bordant to $\lambda \oplus R^{i}$ has necessarily $w_{1}(\eta)=\alpha$ and $w_{r}(\eta)=0$ for $r>1$, where $\alpha \in H^{1}\left(R P(2 n), Z_{2}\right)$ is the generator; on the other hand, one knows from [11] that if $\eta$ is bordant to $T(R P(2 n))$, then $W(\eta)=$ $(1+\alpha)^{2 n+1}$. These facts produce a simultaneous bordism; that is, they imply that, up to permutation, $\left(\bigoplus_{j=1}^{3} \varepsilon_{j}\right) \cup\left(\bigoplus_{j=1}^{3} \mu_{j}\right)$ is bordant to

$$
\left(\left(\lambda \oplus R^{i}\right) \oplus\left(\lambda \oplus R^{j}\right) \oplus T(R P(2 n))\right) \cup\left(R^{2 n+i+1} \oplus R^{2 n+j+1} \oplus 0\right)
$$

as elements of $\bigoplus \mathcal{N}_{p}(B O(r) \times B O(s) \times B O(t))$. In order to complete the classification our next task will consist then in exhibiting a list of $\left(Z_{2}\right)^{2}$-actions realizing all these possibilities. To do this, observe first that from a given involution $(W, T)$ with fixed data $\eta \rightarrow F$ one can construct the following special $\left(Z_{2}\right)^{2}$-actions:

i) $(W \times W ; D, S)$, with $D(x, y)=(T(x), T(y))$ and $S(x, y)=(y, x)$; the fixed data is $\eta \oplus \eta \oplus T(F) \rightarrow F$;

ii) $(W ; T, I d)$, with fixed data $\eta \oplus 0 \oplus 0 \rightarrow F$. 
We remark that the above actions are, respectively, the actions $\Gamma_{2}^{2}(W, T)$ and $\Gamma_{1}^{2}(W, T)$ mentioned in the introduction. Next observe that each automorphism $\sigma:\left(Z_{2}\right)^{2} \rightarrow\left(Z_{2}\right)^{2}$ gives rise, from a given $\left(Z_{2}\right)^{2}$-action $(M, \Phi), \Phi=\left(T_{1}, T_{2}\right)$, to a new action given by $\left(M ; \sigma\left(T_{1}\right), \sigma\left(T_{2}\right)\right)$; we denote this action by $\sigma(M, \Phi)$. When $\sigma$ varies in $\operatorname{Aut}\left(\left(Z_{2}\right)^{2}\right)$, the actions $\sigma(M, \Phi)$ realize all possible permutations of the original fixed data.

Take now $0 \leq i, j \leq k_{n}$ with $i<j$. By applying $j-i$ times Theorem 2 to the fixed data of $\Gamma_{2}^{2} \Gamma^{j}\left(R P(2 n+1), \tau_{0}\right)$ one obtains a $\left(Z_{2}\right)^{2}$-action $\left(N_{i, j}, \varphi_{i, j}\right)$ with fixed data

$$
\left(\left(\lambda \oplus R^{i}\right) \oplus\left(\lambda \oplus R^{j}\right) \oplus T(R P(2 n))\right) \cup\left(R^{2 n+i+1} \oplus R^{2 n+j+1} \oplus 0\right)
$$

(since a $\left(Z_{2}\right)^{2}$-action cannot fix precisely one point $[4,31.3], N_{i, j}$ is necessarily connected; such an action can be explicitly obtained by using the method of [5]).

The following $\left(Z_{2}\right)^{2}$-actions complete then our task:

i) $\sigma \Gamma_{t}^{2} \Gamma^{i}\left(R P(2 n+1), \tau_{0}\right)$, with $0 \leq i \leq k_{n}, t=1$ or 2 and $\sigma \in \operatorname{Aut}\left(\left(Z_{2}\right)^{2}\right)$;

ii) $\sigma\left(N_{i, j}, \varphi_{i, j}\right)$, with $0 \leq i, j \leq k_{n}, i<j$ and $\sigma \in \operatorname{Aut}\left(\left(Z_{2}\right)^{2}\right)$.

Summarizing we obtain

Theorem 3. $A\left(Z_{2}\right)^{2}$-action fixing $R P(2 n) \cup\{p\}$ is necessarily bordant to one of the above actions.

\section{REFERENCES}

1. A. Borel and F. Hirzebruch, On characteristic classes and homogeneous spaces;I, Am. J. Math. 80 (1958), 458-538. MR 21:1586

2. C. Kosniowski and R. E. Stong, Involutions and characteristic numbers, Topology 17 (1978), 309-330. MR 82a:57036

3. D. C. Royster, Involutions fixing the disjoint union of two projective spaces, Indiana Univ. Math. J. 29 (1980), 267-276. MR 81i:57034

4. P. E. Conner and E. E. Floyd, Differentiable Periodic Maps, Springer-Verlag, Berlin, 1964. MR 31:750

5. P. L. Q. Pergher, An equivariant construction, Proc. Amer. Math. Soc. 119 (1993), 319-320. MR 93k:57065

6. P. L. Q. Pergher, Manifolds with $\left(Z_{2}\right)^{k}$-actions, Proc. Amer. Math. Soc. 106 (1989), 10911094. MR 89m:57039

7. P. L. Q. Pergher, The union of a connected manifold and a point as fixed set of commuting involutions, Topology Appl. 69 (1996), 71-81. MR 96m:57055

8. P. L. Q. Pergher, $\left(Z_{2}\right)^{k}$-actions fixing a product of spheres and a point, Canad. Math. Bull. 38 (1995), 366-372. MR 96j:57045

9. R. E. Stong, Bordism and involutions, Ann. of Math. 90 (1969), 47-74. MR 39:3503

10. R. E. Stong, Equivariant bordism and $\left(Z_{2}\right)^{k}$-actions, Duke Math. J. 37 (1970), 779-785. MR 42:6847

11. R. E. Stong, Involutions fixing projective spaces, Michigan Math. J. 13 (1966), 445-447. MR 34:6795

Universidade Federal de São Carlos, Departamento de Matemática, Rodovia Washington Luiz, KM. 235, 13.565-905, SÃo Carlos, S.P., Brazil

E-mail address: pergher@power.ufscar.br 